\title{
MRNA-derived KRAS-targeted Vaccine V941
}

National Cancer Institute

\section{Source}

National Cancer Institute. MRNA-derived KRAS-targeted Vaccine V941. NCI Thesaurus. Code C162186.

A lipid nanoparticle (LNP)-formulated mRNA-based cancer vaccine that targets four of the most commonly occurring KRAS mutations (G12D, G12V, G13D, and G12C), with potential immunostimulatory and antineoplastic activities. Upon vaccination, the mRNAderived KRAS-targeted vaccine V941 (mRNA-5671) is taken up and translated by antigen presenting cells (APCs). Following translation, the epitopes are presented via major histocompatibility complex (MHC) molecules on the surface of the APCs. This leads to an induction of both cytotoxic T-lymphocyte (CTL)- and memory $\mathrm{T}$-cell-dependent immune responses that specifically target and destroy tumor cells harboring these specific KRAS mutations. KRAS, a tumor-associated antigen (TAA), is mutated in a variety of tumor cell types. It plays a key role in tumor cell proliferation and survival and is associated with tumor initiation, metastasis and poor prognosis. 